\title{
CULTURAL VALUES INFLUENCE ON THE ADOPTION OF PRIVACY THREATENING SOCIAL NETWORKS FEATURES
}

\author{
Francesc Miralles, Rosa Rudo and Ferran Giones \\ La Salle, Ramon Llull University, C/Llucanes, 41, Barcelona, Spain
}

Keywords: $\quad$ Social Networks, IT Adoption, IT User Acceptance, Cultural Values, Privacy Concerns.

\begin{abstract}
The effect of cultural values in IT adoption has attracted growing interest in the last years. Researchers posit that cultural values can shed some additional light on the factors that determine IT user acceptance and use. In this research in progress work, the authors propose a model based on previous user acceptance theories to develop a research study to inquire the role that individual cultural values play on the adoption of those social networks features that threat user's privacy the most. What the authors posit is that adoption of those features that are more critical from the point of view of users' privacy can be explained from the perspective of individual's cultural values. In this preliminary work, the authors have developed the model and have drawn a set of hypotheses. In the following steps of the research the authors are going to develop a survey to start the quantitative research.
\end{abstract}

\section{INTRODUCTION}

The emergence of social networking sites is producing a massive impact in the Internet usage (Fraser \& Dutta, 2008). Despite the fast-growing levels of social networks user adoption, it remains to be seen, how individual privacy expectations are adjusted. The aim of this research in progress is to investigate the following research question: Do individual's cultural values explain the adoption of privacy threatening features in social networks?

The paper, therefore, builds on both, the theoretical contribution of IT adoption literature and on the role of cultural values in IT adoption. The model integrates the UTAUT model (Venkatesh et al., 2003), and the Open Cultural Orientation construct (Hwang, 2004). Additionally, ad-hoc variables relevant in social networks research such as attitude (Venkatesh et al., 2003) and trust (Mayer, Davis \& Schoorman, 1995) have been incorporated in the model.

In order to study the research question, the authors expect to issue a quantitative research based on a structural equation model (SEM). In this paper, the authors propose the model to be validated in the quantitative research.

This paper is structured as follows. First, section 2 presents the literature review on social networks,
IT adoption and cultural values frameworks. Next, section 3 describes the research model. Finally, section 4 provides the further steps in this research project.

\section{LITERATURE REVIEW}

\subsection{Social Networks}

A social network is a concept which can be defined as a group of nodes tied with social relationships. If this concept is focused on Web 2.0, social networks are a technology which allows people to connect, interact, work and share opinions, videos,... (Fraser \& Dutta, 2008).

Social networks users disclose all kind of personal information, involuntarily allowing third parties to track and study their behavior (Gross \& Acquisti, 2005), lack of controls on private information exposure has severely threatened users privacy.

\subsection{Acceptance Theories}

Based on previous models of user acceptance of information technology, Venkatesh et al. (2003) proposed a unified model for user acceptance of IT. 


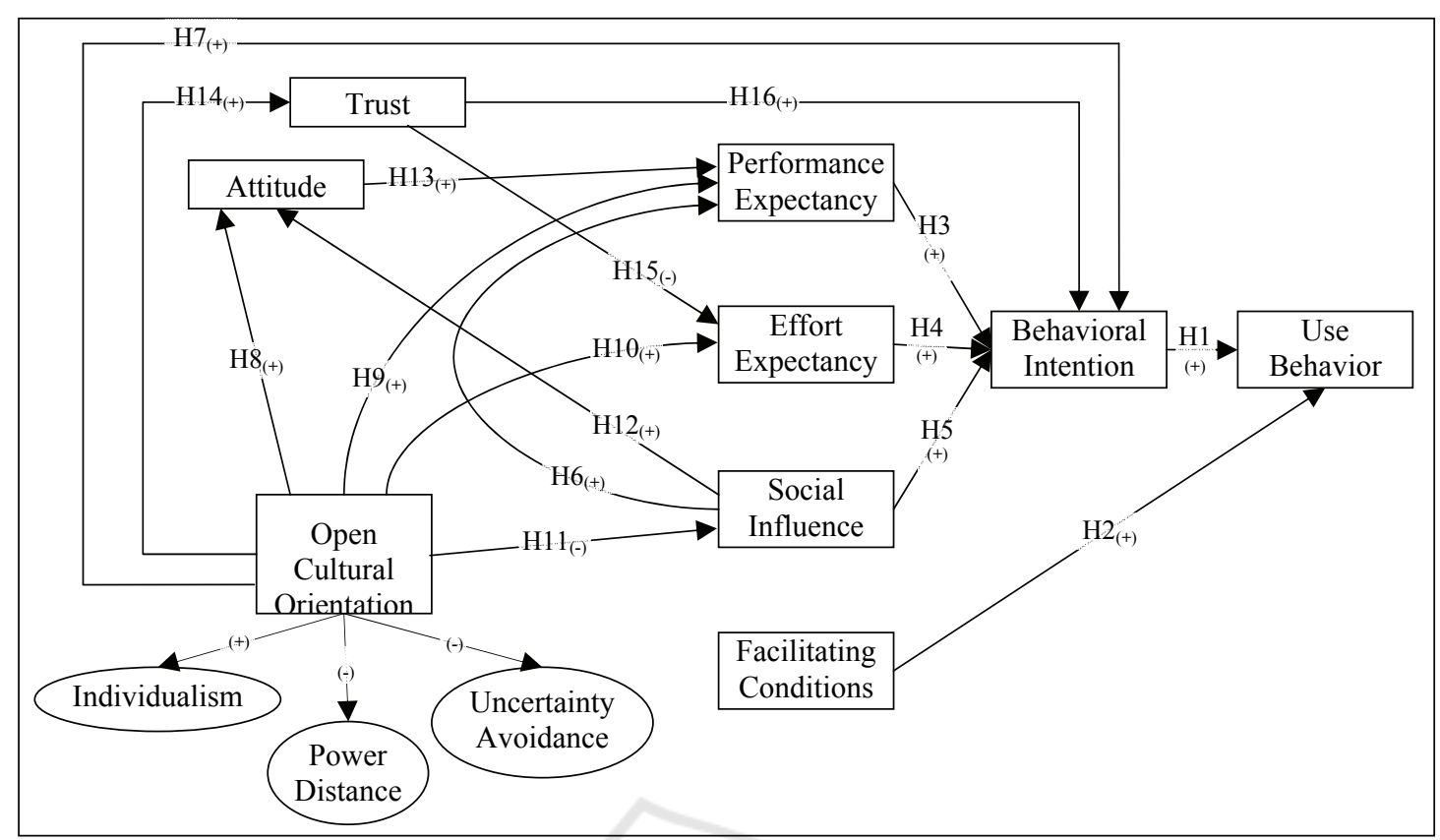

Figure 1: Research Model.

The model was called the Unified Theory of Acceptance and Use of Technology (UTAUT) and included most of the items that were proposed by the previous models.

The model for this research-in-progress work is based on the UTAUT with some additional items to include the effect of individual cultural values. Based on Dorfman \& Howell (1988), and Hwang (2004) an Open Cultural Orientation construct is used to reflect these individual cultural values in the UTAUT model.

\subsection{Culture Values and IT Uses}

Culture values have been a persistent worry to management researchers (Tsui \& Nifadkar, 2007).

IT adoption and diffusion is one of the areas where cultural values have been used the most (Leidner \& Kayworth, 2006). A majority of these studies were based on cultural values at a national level (Hofstede \& Hofstede, 2005). In this research in progress work the authors focus on the individual cultural values and their goal is to shed some light on the effects of culture on the usage of social networks.

\section{RESEARCH MODEL}

The research model is depicted in Figure 1. The model proposes to combine UTAUT (Venkatesh et al., 2003), the Open Cultural Orientation construct (Dorfman \& Howell, 1988), and the social network characteristic use (Fraser \& Dutta, 2008) to study individual privacy concern levels from the perspective of IT adoption and cultural values.

The presented conceptual model has been enriched with the data obtained via semi-structured personal interviews with social network users. The participants are last year students in Telecommunications and IT Management programs.

The interviews provided qualitative data to enrich our model variables and to support the hypothesis formulation based on proposed causal relationships.

As in most of the IT acceptance models the dependent variable is based on use behavior. In this model, Use Behavior depicts the different levels of use based on users' privacy concerns. Two levels have been identified: 'high privacy concern usage' and 'low privacy concern usage'. 'High privacy concern usage' includes those users that take into account the impact of the shared information in their personal privacy. In contrast, 'low privacy concern usage' would contain profiles whose participation in social networks is not affected by a certain degree of self control on private information sharing.

In the following sections of this chapter, there is a detailed description of the variables that build up the model and the hypotheses that have been formulated from the model. 


\subsection{User Acceptance Constructs}

\subsubsection{Social Networks Use Behavior}

As presented in the model, Use Behavior is determined by Behavioral Intention of use. In the interviews conducted, Behavioral Intention was observed in both the 'high privacy concern usage' and 'low privacy concern usage' profiles; as 'low privacy concern usage' profiles foresee to do a more intensive use while 'high privacy concern usage' profiles expect to get more involved in the network activity. Hence, the authors propose that:

$\mathrm{H} 1$ : Intention to use social networks is positively related to 'low privacy concern usage'.

When asked about the devices being used to access the social networks most of the respondents mentioned that they would connect using their home $\mathrm{PC}$, also being interested in using other new access devices such as cell phones. Giving support to the idea that as device connectivity improves, lower levels of privacy concern could be expected. Therefore the authors propose that:

$\mathrm{H} 2$ : Facilitating Conditions are positively related to 'low privacy concern usage'.

\subsubsection{Social Networks Intention of Use}

Performance Expectancy. Social networks Performance Expectancy is based on the perception of the influence that the usage of social networks can have to improve social relationships. Participants expressed their perception that social networks use would derivate in better social relations, quoting one of the respondents "...they help to sustain, improve and make new relationships with different type of people...". In general consistent social relationship improvement expectancy was observed; accordingly, the authors introduce the following hypothesis:

H3: Social Relationship Improvement Expectancy is positively related to social network Behavioral Intention.

Effort Expectancy. Effort Expectancy introduced in TAM (Davis, 1989) as Perceived Ease of Use, directly relates to the individual's intention to use. Higher intention of Use Behavior would be expected if there is a lower perceived effort in using the technology, therefore, this leads to the following hypothesis:

H4: Perceived Ease of Use is positively related to social network Behavioral Intention.

Social Influence. Social influence on IT user acceptance models is related to user's perception about others' opinion on using this technology. Social networks play a relevant role in fostering social relationships (Fraser \& Dutta, 2008). Social networks, by definition, have an inherent relation to social influence, it seems coherent that if others perceive that being in a social network can be positive, the individual would have higher use behavior intention. As some of the respondents contextualize: "...as everyone has it, and to be in touch with my friends I created a profile...".

Consequently, the authors propose the following hypotheses:

H5: Other's Favourable Opinion on using Social Networks is positively related to social network Behavioral Intention.

H6: Other's Favourable Opinion on using social networks is positively related to Social Relationship Improvement Expectancy.

\subsection{Open Cultural Orientation}

Individual Open Cultural Orientation is defined by high individualism, low power distance and low uncertainty avoidance (Hwang, 2004).

Participants in the interviews have reported different values in their attitudes towards the variables that define the Open Cultural Orientation construct. Observed outcomes related to the individualism variable would include participants' expressions such as: "...I prefer to work with other people, as better ideas come out..." or "...I rather prefer to work alone...".

It is expected a positive correlation of Open Cultural Orientation with Social Relationship Improvement Expectancy, Ease of Use and Attitude, but negative correlation with Others' Favourable Opinion as it is expected less influence of Bandwagon effect in high Open Cultural Orientation individuals. Therefore, the authors propose:

H7: High Individual Open Cultural Orientation is positively related to social network Behavioral Intention.

H8: High Individual Open Cultural Orientation is positively related to Attitude.

H9: High Individual Open Cultural Orientation is positively related to Social Relationship Improvement Expectancy.

H10: High Individual Open Cultural Orientation is positively related to Perceived Ease of Use

H11: High Individual Open Cultural Orientation is negatively related to influence of Others' Favourable Opinion on using social networks. 


\subsection{Ad-hoc Variables}

\subsubsection{Attitude Construct in Social Networks}

Social networks have combined their basic applications, with other functionalities that reinforce the attitude of their usage (Venkatesh et al., 2003). As one of the participants described: "...the time spent in social networks is enjoyable and fun...". Therefore, it is expected that Attitude influences directly the Social Relationship Improvement Expectancy, but it is also expected Others' Favourable Opinion to directly drive use Attitude. Hence, the authors hypothesize:

H12: Others' Favourable Opinion on using social networks is positively related to Attitude.

H13: Attitude on social networks is positively related to Social Relationship Improvement Expectancy.

\subsubsection{Trust Construct in Social Networks}

Trust is defined as "the willingness of a party to be vulnerable to the actions of another party based on the expectation that the other will perform a particular action important to the trustor, irrespective of the ability to monitor or control that other party" (Mayer, Davis \& Schoorman, 1995).

Based on respondents answers it is expected that Trust will be related to Behavioral Intention, thus high levels of Trust could encourage high Behavioral Intention. Furthermore, Trust could improve Perceived Ease of Use. It also expected that trust will be influenced by individual Open Cultural Orientation. Hence, the authors propose the following hypotheses:

H14: High Individual Open Cultural Orientation is positively related to Trust.

H15: Trust is negatively related to Perceived Ease of Use.

H16: Trust is positively related to social networks Behavioral Intention.

\section{SUMMARY AND NEXT STEPS}

This research studies the influence of individual cultural values in the use behavior of social networks. In this position paper, the authors propose a model that hypothesizes a causal relationship between individual cultural values and the use of social networks privacy threatening features. The model is built up from previous theories and has been completed using information from social networks users' interviews.

In the following steps of this research project, the authors aim to design and build a questionnaire to validate the model hypotheses. A structural equation model (SEM) is going to be issued to assess the support for the causal relationship between the model variables.

\section{REFERENCES}

Davis, F.D. 1989, "Perceived usefulness, perceived ease of use, and user acceptance of information technology", MIS Quarterly, vol. 13, no. 3, pp. 319-340.

Dorfman, P.W. \& Howell, J.P. 1988, "Dimensions of national culture and effective leadership patterns: Hofstede revisited", Advances in international comparative management, vol. 3, pp. 127-150.

Fraser, M. \& Dutta, S. 2008, Throwing sheep in the Boardroom. How Online Social Networking Will Transform Your Life, Work and World, 1st Ed, Wiley, Chichester, England.

Gross, R. \& Acquisti, A. 2005, "Information revelation and privacy in online social networks", Proc. of the 2005 ACM Workshop on Privacy in the electronic society, Alexandria, VA, USA, Nov., 2005.

Hofstede, G. \& Hofstede, G.J. 2005, Cultures and Organizations: Software of the Mind, 2nd Ed, McGraw-Hill, New York, USA.

Hwang, Y. 2004, "The Influence of Individual-level Cultural Orientation on ERP System Adoption", AMCIS 2004 Proc., New York, August, 5-8 2004.

Leidner, D.E. \& Kayworth, T. 2006, "A review of culture in information systems research: Toward a theory of information technology culture conflict", Management Information Systems Quarterly, vol. 30, no. 2, pp. 9.

Mayer, R.C., Davis, J.H. \& Schoorman, F.D. 1995, “An integrative model of organization trust", The Academy of Management Review, vol. 20, no. 3, pp. 709-734.

Tsui, A. S. \& Nifadkar, S.S. 2007, "Cross-national, crosscultural organizational behavior research: Advances, gaps, and recommendations", Journal of management, vol. 33, no. 3, pp. 426.

Venkatesh, V., Morris, M.G., Davis, G.B. \& Davis, F.D. 2003, "User acceptance of information techonolgy: Toward a unified view", Mis Quarterly, vol. 27., no. 3, pp. 425-478. 